2020 and 11/01/2021 and included questions relating to preCOVID ECT (June 2019 to February 2020) and during-COVID ECT (March 2020 to November 2020). Questions were around travel of staff/patients for ECT, whether they had a preference for a model and their reasons around it. Data were summarised in MS Excel and free text comments analysed to gain an understanding of staff s preferences and reasoning behind their choices. Result. Although some boroughs had patients attending from other boroughs in pre-COVID times, considerably more staff (53.85\%) and patients (61.54\%) had to travel for ECT during COVID times. Around 50\% staff expressed a clear view for decentralised services; the common reasons being safer for patients, better continuity of care, less travelling issues, patients more likely to consent, easier to manage correct paperwork, easier to send staff who knows patients well, less driving for staff and likely less cancellations. Around $40 \%$ staff expressed a clear view for centralised services; the common reasons being less staff needed, better infection control, easier to maintain staff skills, efficiency, developing clinical expertise with larger number of cases, education opportunities, better set-up of clinics, transportation and accessibility. Some concerns raised for the centralised model were managing patients with complex anaesthesia, travelling for unwell/ disturbed patients, too high patient numbers, poor communication and impact on training.

Conclusion. In summary, there was a mixed view of which services are preferable. Further discussion in trust wide ECT forum will be helpful to move things forward. Although it is likely that services may shift from a decentralised to a centralised system, we need to ensure this is done safely and in particular, address the main concerns around centralisation.

\section{Preliminary investigation into the identification and management of catatonia in patients admitted to adult inpatient units}

Joanna Moore ${ }^{1 \star}$, Amy Kunicki², Georgina Latcham², Eleanor Perkins ${ }^{2}$ and Emma Vaccari ${ }^{2}$

${ }^{1}$ Dorset Healthcare University NHS Foundation Trust and ${ }^{2}$ Southern Health NHS Foundation Trust

${ }^{*}$ Corresponding author.

doi: 10.1192/bjo.2021.881

Aims. The prevalence of catatonia is considered to be approximately $10 \%$ in psychiatric inpatients. Clinical experience suggests a lower documented prevalence. This could cause longer admissions and complications, such as Neuroleptic Malignant Syndrome (NMS). We carried out a service evaluation to investigate the recognition and management of catatonia on inpatient units in Southern Health Foundation Trust (SHFT). We reviewed the local documented prevalence of catatonia, treatment offered and prevalence of complications.

Method. We retrospectively reviewed the electronic records of 95 consecutive admissions to four adult inpatient units in SHFT, starting on 1st August 2020. We reviewed notes for the admission to establish whether catatonia was suspected and identified. We applied the screening questions from the Bush-Francis Catatonia Rating Scale (BFCRS) to the documented mental state examinations (MSE) prior to, and shortly after, admission. We also recorded the prescriptions issued during the first 72 hours of admission, and whether patients developed neuroleptic malignant syndrome (NMS), serotonin syndrome or required admission to a general hospital during admission.

Result. Catatonia was documented as a possibility for 2 patients (2.1\%). One showed possible posturing and stupor, while there were no documented symptoms for the other. In both cases the possibility was discounted by the clinical team. Twelve patients (12.6\%) showed one or more possible or confirmed signs of catatonia. Eleven of these were prescribed regular antipsychotic medication on admission, but only 3 were prescribed regular benzodiazepines. NMS was more likely to be suspected in patients with a BFCRS of 1 or more compared with those with a score of 0 , with an odds ratio of 8.1 (95\% CI [1.03-64.0], Fisher's exact test = $7.79, \mathrm{p}=.076$ ).

Conclusion. Catatonia is likely under-recognised and undertreated locally among psychiatric inpatients. Although only approaching statistical significance, the higher rate of suspected NMS in patients showing possible catatonia is noteworthy and needs further investigation. Regular benzodiazepines were not frequently prescribed in this group, while antipsychotics, prescribed in all of these patients, can precipitate NMS. Alternatively, this finding could reflect the overlap in clinical presentation between NMS and catatonia. Data collection was limited by the frequent use of "remote clerking", in the context of the COVID-19 pandemic. Additionally, the quality of mental state examinations was often not sufficient to draw any conclusions on the possible presence or absence of catatonic symptoms. This project has highlighted practice in need of improvement, which will be further prospectively investigated and improved via a Quality Improvement Project.

\section{Physical health monitoring of patients prescribed depot antipsychotic medication in north west Edinburgh community mental health team}

Douglas Murdie $^{1 \star}$, Jakub Wojtowicz ${ }^{2}$, Alexandra Thompson ${ }^{2}$, Anne MacLeod ${ }^{2}$, Adam Mallis ${ }^{2}$, Hamsi Evans², Joshua Haggart ${ }^{2}$, Hae Choi $^{2}$ and Vikki Argent ${ }^{3}$

${ }^{1}$ Consultant Psychiatrist, NW CMHT, NHS Lothian; ${ }^{2}$ University of Edinburgh Medical School - 1st year Medical Student and ${ }^{3}$ Consultant Perinatal Psychiatrist, Perinatal MHT, NHS Lothian ${ }^{\star}$ Corresponding author.

doi: 10.1192/bjo.2021.882

Aims. To monitor the quality of physical health monitoring of patients prescribed depot antipsychotic medication in the North West Edinburgh Community Mental Health Team (CMHT). We also evaluated the completeness of prescriptions and Mental Health Act (Scotland) (Act) 2003 paperwork where relevant.

Background. Antipsychotic medications are medicines for treating conditions such as Schizophrenia, but some may be associated with an increased risk of Metabolic Syndrome. Moreover, evidence indicates that patients with major mental disorder have a reduced life expectancy in comparison to those without such diagnoses. These two factors illustrate the importance of the physical health of this patient cohort being monitored on a regular basis. This project will evaluate how a local CMHT is performing, with the possibility of enacting service improvements if required.

Method. The records of the 60 patients prescribed depot antipsychotic medication administered by this CMHT were reviewed. A check-list was created consisting of 14 categories analysing the quality of physical health monitoring, as well as compliance with prescription standards and, where relevant, Mental Health Act (Scotland) (Act) 2003 paperwork. We compared patient records against our checklist for the calendar year of 2019. The Scottish Intercollegiate Guidelines Network (SIGN) 131 (Management of Schizophrenia) section 5.2 was used as the gold standard for physical health monitoring against which the data we collected was compared. 
Result. We identified a wide range of flaws with the current system and implementation of monitoring, and difficulty in locating the required information. There was no consistent monitoring of physical observations on electronic record, nor an accepted alternative way in which this was documented. Furthermore, blood tests were not consistently obtained either by the service or GP practices in a reproducible manner. This led to discussions within the CMHT regarding creation of a new pathway for the monitoring of this patient cohort using a Quality Improvement model, with the ultimate goal to establish a regular physical health clinic.

Conclusion. There is significant evidence that patients with major mental disorder do not access healthcare as consistently as those without, leading to a disparity in life expectancy. In light of the fact that antipsychotic medications can be associated with Metabolic Syndrome, we have an even greater responsibility to tackle this marked health inequality by appropriately monitoring our patients. This was not done well in this particular CMHT, but this project will lead to improvements in the service and ultimately patient care.

Does the advice requested by carers of people who live with dementia reflect the level of commissioned post-diagnostic support? A retrospective evaluation of calls to the Me2U dementia day centre 24-hour advice line

Rajan Nathan ${ }^{1 \star}$, Stephen Callaghan ${ }^{2}$, Kelly Walker ${ }^{3}$, Angela Mason $^{3}$ and Rosemarie Whittington ${ }^{3}$

${ }^{1}$ Cheshire and Wirral Partnership NHS Foundation Trust; ${ }^{2} \mathrm{EQE}$ Health Ltd and ${ }^{3} \mathrm{Me} 2 \mathrm{u}$ Dementia Day Centre

${ }^{\star}$ Corresponding author.

doi: 10.1192/bjo.2021.884

Aims. The aim was to examine the reasons for advice requests by carers of people who live with dementia (PLWD) that attend the $\mathrm{Me} 2 \mathrm{u}$ dementia day centre in order to identify key explanatory themes. We hypothesised that requests were related mainly to coordinating care and clinical issues due to limited postdiagnostic support (PDS) in our area.

Background. The Me2u dementia day centre (Merseyside) cares for PLWD and also supports carers. As part of the service, a 24-hour advice line is included for PLWD and their carers who attend the centre. Locally, there is limited PDS and most carers navigate the health and social care system alone mirroring the findings by the National Collaborating Centre for Mental Health (NCCMH).

Method. We undertook a retrospective evaluation of 244 advice calls, from 64 carers, between 01/06/2019 and 31/12/2019. We analysed time of call, type of advice, type of dementia, age and whether the advice was for the PLWD or for the carer.

Result. Of the 244 calls, the most common time to call was between $09.00-14.00(\mathrm{n}=168$; $(68.8 \%)$ peak $09.00-10.00(\mathrm{n}=$ 38). Average age of the person about whom the advice was sought was 79.08 years. $91.4 \%$ of the advice calls related to PLWD (most common dementia Alzheimer's) and $8.6 \%$ to the carer only. The mean number of calls per person was 3.8 (range 1-24).

Advice data were grouped into 9 broad themes namely, related to symptoms/behaviour $(32.79 \%, \mathrm{n}=80)$, request for $\mathrm{Me} 2 \mathrm{u}$ to coordinate care $(20.08 \%, \mathrm{n}=49)$, general advice $(14.75 \%, \mathrm{n}=36)$, personal care $(9.42 \%, \mathrm{n}=23)$, carer only advice $(8.60 \%, \mathrm{n}=21)$, social issues $(6.14 \%, \mathrm{n}=15)$, social care $(4.50 \%, \mathrm{n}=11)$, safeguarding $(2.46 \%, n=6)$, non-health and social care issue $(1.23 \%, n=3)$. Conclusion. Reasons for limited/poor PDS given by the NCCMH are; absence of named coordinators of care, over-reliance on families and carers to manage and facilitate appointments, poor recognition and management of comorbidities. This data show that $52.87 \%$ of calls were for clinical advice and coordination of care reflecting NCCMH findings. The interventions post-call reduced the impact on providers of urgent care.

These findings provide support for the provision of a [24-hour] advice line as a routine part of post-diagnostic support services, especially in areas that have limited or poor PDS. Commissioners of PDS services in areas that have limited or poor PDS should make this a priority to prevent unplanned admissions to hospital and carer breakdown.

\section{Vitamin D monitoring and management within men's} secure services

Jason Niblett ${ }^{1 \star}$, Shay-Anne Pantall ${ }^{2}$ and Anis Ahmed ${ }^{2}$

${ }^{1}$ University of Birmingham, Medical School and ${ }^{2}$ Birmingham and Solihull Mental Health NHS Foundation Trust

${ }^{*}$ Corresponding author.

doi: 10.1192/bjo.2021.885

Aims. To audit the investigation, identification and treatment of Vitamin D deficiency within Men's Secure Services.

Background. Vitamin D and/or vitamin D deficiency has been suggested to play a role in the pathogenesis of mental illness. There is evidence that Vitamin D inadequacy is pandemic among rehabilitation patients in inpatient settings. Patients within secure hospitals are similarly considered to be at high risk, due to their limited solar exposure during often lengthy admissions. It has been suggested that these patients should be considered an 'at-risk' cohort, for whom Vitamin D supplementation should be routine. Men's secure services in Birmingham comprise of two medium secure units and a low secure rehabilitation unit. Here we present an audit of Vitamin D monitoring and treatment completed in 2019. Method. A three year retrospective review of electronic patient records, for all inpatients admitted within men's secure services as of 1 September $2019(n=188)$. Standards were based on the Trust accepted guidelines for management of Vitamin D deficiency. Result. Key findings included:-

The majority of inpatients were Caucasian (43\%) and AfricanCaribbean (24\%). Ages ranged from 18 to 70 , with a mean age of 39. Approximately two-thirds (65\%) had been in hospital for over a year, of which $44 \%$ had been admitted for more than 3 years. The average length of admission was 885 days.

Only $47 \%$ of patients had their Vitamin D level checked within the study period.

Of those checked, $24 \%$ were tested within 1 month of admission. The mean duration between admission and Vitamin D testing was 464 days.

Results ranged from 10.3 to $118.5 \mathrm{nmol} / \mathrm{L}$. A high rate of Vitamin D deficiency was identified (54\%), whilst a further $16 \%$ had 'inadequate' levels.

$23 \%$ of those identified as requiring treatment did not receive any supplementation, whilst $59 \%$ of those with sufficient Vitamin D were prescribed treatment.

Only $48 \%$ had their levels rechecked following treatment; of these, only $59 \%$ now had an adequate Vitamin D status.

Conclusion. This audit demonstrates limited Vitamin D monitoring within male forensic inpatients. There was a high prevalence of Vitamin D insufficiency in this population, yet a substantial proportion of patients with identified deficiency were not prescribed any treatment. Ongoing monitoring and review of 